KRL-MAP-283

\title{
What Do We Know About the Strange Magnetic Radius?
}

\author{
H.-W. Hammer ${ }^{a}$, S.J. Puglia ${ }^{a}$, M.J. Ramsey-Musolf ${ }^{b, c}$, and Shi-Lin Zhu ${ }^{b}$ \\ a Department of Physics, The Ohio State University, Columbus, OH 43210 USA \\ ${ }^{b}$ California Institute of Technology, Pasadena, CA 91125 USA \\ ${ }^{c}$ Department of Physics, University of Connecticut, Storrs, CT 06269 USA
}

\begin{abstract}
We analyze the $q^{2}$-dependence of the strange magnetic form factor, $G_{M}^{(s)}\left(q^{2}\right)$, using heavy baryon chiral perturbation theory $(\mathrm{HB} \chi \mathrm{PT})$ and dispersion relations. We find that in $\mathrm{HB} \chi \mathrm{PT}$ a significant cancellation occurs between the $\mathcal{O}\left(p^{2}\right)$ and $\mathcal{O}\left(p^{3}\right)$ loop contributions. Consequently, the slope of $G_{M}^{(s)}$ at the origin displays an enhanced sensitivity to an unknown $\mathcal{O}\left(p^{3}\right)$ low-energy constant. Using dispersion theory, we estimate the magnitude of this constant, show that it may have a natural size, and conclude that the low- $q^{2}$ behavior of $G_{M}^{(s)}$ could be dominated by nonperturbative physics. We also discuss the implications for the interpretation of parity-violating electron scattering measurements used to measure $G_{M}^{(s)}\left(q^{2}\right)$.
\end{abstract}


The flavor structure of low-energy nucleon properties (e.g., mass, spin, etc.) continues to be a subject of considerable interest in hadron physics. In particular, evidence exists that strange quarks may play a non-negligible role in such low-energy properties [四]. The strange quark contribution to the nucleon's electromagnetic structure is under investigation using parity-violating electron scattering experiments at MIT-Bates [2], the Jefferson Lab (JLab) [3, [4], and Mainz [5]. These experiments have produced the first determinations of the strange magnetic form factor, $G_{M}^{(s)}\left(q^{2}\right)$ [2], as well as a linear combination of $G_{M}^{(s)}\left(q^{2}\right)$ and the strange electric form factor, $G_{E}^{(s)}\left(q^{2}\right)$ [3].

Theoretical interest has focused largely on the leading moments of these form factors: the strange magnetic moment, $\mu_{s}=G_{M}^{(s)}(0)$ and the corresponding electric and magnetic radii, $\left\langle r_{s}^{2}\right\rangle_{E, M}=6 d G_{E, M}^{(s)}\left(q^{2}\right) / d q^{2}$ at $q^{2}=0$ (note that we do not normalize the radii to the $q^{2}=0$ values of the form factors). While the radii are intrinsically interesting, knowledge of $\left\langle r_{s}^{2}\right\rangle_{M}$ is also needed in order to extract $\mu_{s}$ from experiment, since $G_{M}^{(s)}$ can be determined experimentally only for non-zero $q^{2}$. In order to extrapolate $G_{M}^{(s)}$ to the photon point, one might hope to rely on chiral perturbation theory, which is in principle well-suited to characterizing the leading $q^{2}$-dependence of form factors. While $\left\langle r_{s}^{2}\right\rangle_{M}$ is nominally of chiral order $p^{3}$ (we count the magnetic moment operator as being $\mathcal{O}(p)$ ), the leading order contribution from kaon loops is proportional to $1 / m_{K} \sim 1 / \sqrt{m_{s}}$ and, thus, of $\mathcal{O}\left(p^{2}\right)$. At this order, there exist no analytic (in quark mass) operators and, consequently, no counterterm or corresponding low-energy constant. Thus, as observed by the authors of Ref. [G], one is able to make a parameter-free prediction for $\left\langle r_{s}^{2}\right\rangle_{M}$ at this order which may be used to perform a model-independent extrapolation of $G_{M}^{(s)}$ to the photon point. This procedure has been used to extract a value of $\mu_{s}=0.01 \pm 0.29 \pm 0.31 \pm 0.07$ from the value of the form factor obtained by the SAMPLE Collaboration at $q^{2}=-0.1(\mathrm{GeV} / c)^{2}: G_{M}^{(s)}\left(q^{2}=-0.1\right)=0.14 \pm 0.29 \pm 0.31$ [2] (The last error in $\mu_{s}$ corresponds to the theoretical extrapolation uncertainty quoted in Ref. [6].)

Here, we compute the $\mathcal{O}\left(p^{3}\right)$ loop contributions to $G_{M}^{(s)}\left(q^{2}\right)$ in heavy baryon chiral perturbation theory $(\mathrm{HB} \chi \mathrm{PT})$. We show that the $\mathcal{O}\left(p^{3}\right)$ contributions to $\left\langle r_{s}^{2}\right\rangle_{M}$ largely cancel the $\mathcal{O}\left(p^{2}\right)$ term. In addition, we observe that a new magnetic radius operator arises at this order, whose coefficient cannot presently be determined apart from a determination of $G_{M}^{(s)}$ itself. Because of the cancellation between the $\mathcal{O}\left(p^{2}\right)$ and $\mathcal{O}\left(p^{3}\right)$ terms, the relative importance of this coefficient, or low-energy constant (LEC), is enhanced over what one might otherwise expect. Using a dispersion theory analysis of $G_{M}^{(s)}$, we estimate this LEC and argue that it could give the dominant contribution to $\left\langle r_{s}^{2}\right\rangle_{M}$. As a corollary, we suggest that the low-momentum behavior of $G_{M}^{(s)}$ is governed by non-perturbative physics and observe that one cannot presently extract $\mu_{s}$ from experiment in a model-independent manner.

In $\mathrm{HB} \chi \mathrm{PT}$, the contributions to $G_{M}^{(s)}\left(q^{2}\right)$ through $\mathcal{O}\left(p^{3}\right)$ are generated by the graphs of Fig. 1. The $\mathcal{O}(p)$ contributions to $\mu_{s}$ arise from insertion of the lowest-order magnetic operator in Fig. 1(a). The $\mathcal{O}\left(p^{2}\right)$ contributions nominally arise from Figs. 1(b-e). However, graphs 1 (c-e) contribute only to the charge form factor, so that the $\mathcal{O}\left(p^{2}\right)$ contributions to $\mu_{s}$ arise entirely from Fig. 1(b). The $\mathcal{O}\left(p^{3}\right)$ contributions to $\mu_{s}$ arise from several sources: insertion of the tree-level magnetic moment operator in Figs. 1(f,g); operators containing two-derivatives (Fig. 1(h)); 1/ $M_{N}$, or "recoil", corrections in Figs. 1(i-k) (denoted by the "X"); and magnetic operators proportional to $m_{s}$ (Fig. 1(l)). We note that the sum of 
Figs. $1(\mathrm{j})$ and $1(\mathrm{k})$ vanishes for $G_{M}^{(s)}$.

Similarly, the leading contribution to the slope of $G_{M}^{(s)}\left(q^{2}\right)$ at the origin also arises from Fig. 1(b) and is $\mathcal{O}\left(p^{2}\right)$. The $\mathcal{O}\left(p^{3}\right)$ contributions to the slope are given by the sum of Fig. 1(h) and 1(i), along with the insertion of the magnetic radius operator in Fig. 1(a). One must also include the dependence of Fig. 1(b) on $v \cdot q=q^{2} / M_{N}$.

The formalism for evaluating these diagrams in $\mathrm{HB} \chi \mathrm{PT}$ has been discussed extensively elsewhere, so we do not give all the relevant formulae here (see, e.g., Ref. [7] and references therein). However, in order to define our normalization for the LEC's we give expressions for the relevant magnetic moment and radius operators:

$$
\begin{aligned}
\mathcal{L}_{1}= & \frac{e b_{0}}{\Lambda_{\chi}} \epsilon_{\mu \nu \rho \sigma} v^{\rho} \operatorname{Tr}\left(\bar{B} S^{\sigma} B\right) Z^{\mu \nu} \\
& +\frac{e}{\Lambda_{\chi}} \epsilon_{\mu \nu \rho \sigma} v^{\rho}\left\{b_{+} \operatorname{Tr}\left(\bar{B} S^{\sigma}\{Q, B\}\right)+b_{-} \operatorname{Tr}\left(\bar{B} S^{\sigma}[Q, B]\right)\right\} F^{\mu \nu} \\
\mathcal{L}_{S B}= & \frac{e}{\Lambda_{\chi}} \epsilon_{\mu \nu \rho \sigma} v^{\rho} F^{\mu \nu}\left\{b_{3} \operatorname{Tr}\left(\bar{B} S^{\sigma}[[Q, B], \mathcal{M}]\right)\right. \\
& +b_{4} \operatorname{Tr}\left(\bar{B} S^{\sigma}\{[Q, B], \mathcal{M}\}\right)+b_{5} \operatorname{Tr}\left(\bar{B} S^{\sigma}[\{Q, B\}, \mathcal{M}]\right) \\
& \left.+b_{6} \operatorname{Tr}\left(\bar{B} S^{\sigma}\{\{Q, B\}, \mathcal{M}\}\right)+b_{7} \operatorname{Tr}\left(\bar{B} S^{\sigma} B\right) \operatorname{Tr}(\mathcal{M} Q)\right\} \\
& +\frac{e b_{8}}{\Lambda_{\chi}} \epsilon_{\mu \nu \rho \sigma} v^{\rho} Z^{\mu \nu} \operatorname{Tr}\left(\bar{B} S^{\sigma} B\right) \operatorname{Tr}(\mathcal{M}) \\
\mathcal{L}_{2}= & \frac{e b_{0}^{r}}{\Lambda_{\chi}^{3}} \epsilon_{\mu \nu \rho \sigma} v^{\rho} \operatorname{Tr}\left(\bar{B} S^{\sigma} B\right) \partial^{2} Z^{\mu \nu} \\
& +\frac{e}{\Lambda_{\chi}} \epsilon_{\mu \nu \rho \sigma} v^{\rho}\left\{b_{+}^{r} \operatorname{Tr}\left(\bar{B} S^{\sigma}\{Q, B\}\right)+b_{-}^{r} \operatorname{Tr}\left(\bar{B} S^{\sigma}[Q, B]\right)\right\} \partial^{2} F^{\mu \nu}
\end{aligned}
$$

where $B$ denotes the baryon field octet, $Q=\operatorname{diag}(2 / 3,-1 / 3,-1 / 3)$ (at lowest order), $\mathcal{M}=\operatorname{diag}(0,0,1)$ is the $\mathrm{SU}(3)$ symmetry-breaking matrix associated with the light quark masses, $v^{\rho}$ and $S^{\sigma}$ are the baryon velocity and spin, respectively, $F^{\mu \nu}$ is the photon field strength tensor, and $Z^{\mu \nu}$ is the corresponding tensor for a source coupling to baryon number current $^{1}$. The chiral scale $\Lambda_{\chi} \equiv 4 \pi F_{\pi}$, with $F_{\pi}=92.4 \mathrm{MeV}$. The full chiral structure of the electromagnetic charge operator is obtained by making the replacement $Q \rightarrow \frac{1}{2}\left(\xi^{\dagger} Q \xi+\xi Q \xi^{\dagger}\right)$, where $\xi=\exp \left(i \tilde{\Pi} / F_{\pi}\right)$ and $\tilde{\Pi}$ is the octet of pseudscalar mesons. Note that the symmetrybreaking operators in $\mathcal{L}_{S B}$ are suppressed by two powers of $p$ relative to $\mathcal{L}_{1}$. Following standard conventions, however, we absorb this suppression into the definition of the constants $b_{3-8}$. Indeed, when $b_{ \pm}$and the octet constants $b_{3-7}$ are determined from fits to octet baryon magnetic moments [7], the latter are generally suppressed by an order of magnitude relative to the former, as one would expect from the $p^{2}$ suppression of $\mathcal{L}_{S B}$.

The two-derivative operators have the structure

$$
\mathcal{L}_{M B}=\frac{4 i}{\Lambda_{\chi}} \epsilon_{\mu \nu \rho \sigma} v^{\rho}\left\{b_{9} \operatorname{Tr}\left(\bar{B} S^{\sigma} A^{\mu}\right) \operatorname{Tr}\left(A^{\nu} B\right)\right.
$$

\footnotetext{
${ }^{1} \mathrm{~A}$ typo appears in Eq. (17) of Ref. [7]. The term proportional to $b_{6}$ should contain a double anti-commutator.
} 


$$
\left.+b_{10} \operatorname{Tr}\left(\bar{B} S^{\sigma}\left[A^{\mu}, A^{\nu}\right] B\right)+b_{11} \operatorname{Tr}\left(\bar{B} S^{\sigma}\left\{A^{\mu}, A^{\nu}\right\} B\right)\right\}
$$

where $A^{\mu}=(i / 2)\left(\xi^{\dagger} \partial^{\mu} \xi-\xi \partial^{\mu} \xi^{\dagger}\right)$.

Using these normalizations, and expanding

$$
G_{M}^{(s)}\left(q^{2}\right)=\mu_{s}+\frac{1}{6}\left\langle r_{s}^{2}\right\rangle_{M} q^{2}+\cdots
$$

we obtain

$$
\begin{aligned}
\mu_{s}= & \left(\frac{2 M_{N}}{\Lambda_{\chi}}\right)\left\{b_{s}\left(1+\sum_{X=\pi, K, \eta}\left(\gamma^{(X)}-\lambda^{(X)}\right) \frac{m_{X}^{2}}{\Lambda_{\chi}^{2}} \ln \frac{m_{X}^{2}}{\mu^{2}}\right)\right. \\
& +\left(5 D^{2}-6 D F+9 F^{2}\right)\left(\frac{\pi}{3} \frac{m_{K}}{\Lambda_{\chi}}-\frac{5}{6} \frac{m_{K}^{2}}{M_{N} \Lambda_{\chi}} \ln \frac{m_{K}^{2}}{\mu^{2}}\right) \\
& \left.+b_{8}-2\left(b_{3}+b_{4}-\frac{1}{3} b_{5}-\frac{1}{3} b_{6}-b_{7}\right)-h \frac{m_{K}^{2}}{\Lambda_{\chi}^{2}} \ln \frac{m_{K}^{2}}{\mu^{2}}\right\} \\
\left\langle r_{s}^{2}\right\rangle_{M}= & -\frac{6}{\Lambda_{\chi}^{2}}\left\{\left(\frac{2 M_{N}}{\Lambda_{\chi}}\right) b_{s}^{r}+\frac{1}{18}\left(5 D^{2}-6 D F+9 F^{2}\right)\left(\frac{\pi M_{N}}{m_{K}}+7 \ln \frac{m_{K}}{\mu}\right)\right. \\
& \left.-\left(\frac{M_{N}}{9 \Lambda_{\chi}}\right) h \ln \frac{m_{K}}{\mu}\right\}
\end{aligned}
$$

where

$$
\begin{aligned}
b_{s} & =b_{0}-2\left[b_{-}-\left(b_{+} / 3\right)\right] \\
b_{s}^{r} & =b_{0}^{r}-2\left[b_{-}^{r}-\left(b_{+}^{r} / 3\right)\right] \\
h & =b_{9}-2 b_{10}+6 b_{11}
\end{aligned}
$$

and where $\mu$ is the subtraction scale associated with the divergence of the Feynman diagrams, $D$ and $F$ are the SU(3) axial current reduced matrix elements, and $\gamma^{(X)}$ and $\lambda^{(X)}$ are known functions of $D$ and $F$ [7]. The dependence of $b_{s}\left(b_{s}^{r}\right)$ on the $\mathrm{SU}(3)$ singlet constant $b_{0}\left(b_{0}^{r}\right)$ and octet constants $b_{ \pm}\left(b_{ \pm}^{r}\right)$, as well as the presence of the $b_{3-8}$ terms in $\mu_{s}$ arise from the group structure of the strange quark vector current:

$$
\bar{s} \gamma_{\mu} s=J_{\mu}^{B}-2 J_{\mu}^{I=0}(E M)
$$

where $J_{\mu}^{B}$ is the $\mathrm{SU}(3)$ singlet, or baryon number, current and $J_{\mu}^{I=0}(E M)$ is the isoscalar electromagnetic (EM) current, an $\mathrm{SU}(3)$ octet operator. Nucleon matrix elements of the latter receive contributions from the $b_{3-7}$ operators in Eq. (2), as evaluated in Ref. [7]. The operators proportional to $b_{0,8}$ and $b_{0}^{r}$ contribute to $\left\langle p\left|J_{\mu}^{B}\right| p\right\rangle$. While values for the octet LEC's $b_{ \pm}, b_{ \pm}^{r}$, and $b_{3-7}$ can be obtained from measured octet baryon magnetic moments, one has no independent determination of the singlet LEC's $b_{0}, b_{0}^{r}$, and $b_{8}$ (apart from measurements of $G_{M}^{(s)}$ itself). Note also that the LEC's are functions of the subtraction scale, $b_{i}=b_{i}(\mu)$ and $b_{s}=b_{s}(\mu)$.

The contributions through $\mathcal{O}\left(p^{2}\right)$ for both $\mu_{s}$ and $\left\langle r_{s}^{2}\right\rangle_{M}$ have been computed in Refs. 6, 8]. In the case of $\left\langle r_{s}^{2}\right\rangle_{M}$, the $\mathcal{O}\left(p^{2}\right)$ contribution contains the factor $M_{N} / m_{K}$. The contributions 
of $\mathcal{O}\left(p^{3}\right)$ have not been computed previously. As a check on our results, we compare with the integrals appearing in the relativistic calculation of the isovector electromagnetic form factors in Ref. [9]. After replacing $m_{\pi}$ by $m_{K}$, we find agreement with the non-analytic contributions through $\mathcal{O}\left(p^{3}\right)$. Note that symmetry-breaking effects which break the degeneracy of the pseudoscalar decay constants $\left(F_{\pi} \neq F_{K} \neq F_{\eta}\right)$ contribute at higher order than we consider here, so we use only $F_{\pi}$ in our numerical evaluation below. Similarly, the lowest-order effect of the $M_{\Lambda, \Sigma}$ and $M_{N}$ non-degeneracy is analytic in $m_{s}$ and can be absorbed entirely in the LEC's $b_{0}, b_{ \pm}, b_{0}^{s}$, etc.. Finally, as in Ref. [6], we have included only octet baryon intermediate states. While inclusion of decuplet states will likely affect the precise numerical values of the $\mathcal{O}\left(p^{2}\right)$ and $\mathcal{O}\left(p^{3}\right)$ contributions, we do not expect modification of our qualitative conclusions.

The low- $\left|q^{2}\right|$ behavior of $G_{M}^{(s)}$ depends on several LEC's, including $b_{s}, b_{s}^{r}$, and $b_{8-11}$. To determine the relative importance of the LEC's and loops, we take $D=0.75$ and $F=0.5$, set $\mu=1 \mathrm{GeV}$, and derive values for $b_{3} \ldots b_{7}$ from the octet magnetic moments. In doing so, we evaluate the combination of constants $h$ as in Ref. [10]. The result gives

$$
\begin{aligned}
\mu_{s} & =2.5\left[b_{s}(\mu=1 \mathrm{GeV})+0.6 b_{8}(\mu=1 \mathrm{GeV})\right]+1.2 \\
\left\langle r_{s}^{2}\right\rangle_{M} & =-\left[0.04+0.3 b_{s}^{r}(\mu=1 \mathrm{GeV})\right] \mathrm{fm}^{2},
\end{aligned}
$$

where the LEC-independent contributions $1.2\left(\right.$ in $\left.\mu_{s}\right)$ and $-0.04 \mathrm{fm}^{2}\left(\right.$ in $\left.\left\langle r_{s}^{2}\right\rangle_{M}\right)$ arise entirely from the loop graphs and symmetry-breaking LEC's. We note in particular the relative sensitivity of $\left\langle r_{s}^{2}\right\rangle_{M}$ to loops and the LEC $b_{s}^{r}$. Were the latter to have a "natural" size, $\left|b_{s}^{r}\right| \sim 1$, its contribution would dominate the slope of $G_{M}^{(s)}$ and the origin. Indeed, an uncertainty in this LEC of $\Delta b_{s}^{r}= \pm 1$ corresponds to a $\pm 0.3 \mathrm{fm}^{2}$ uncertainty in the radius. This situation contrasts starkly with the result at $\mathcal{O}\left(p^{2}\right)$, which gives $\left\langle r_{s}^{2}\right\rangle_{M}=-0.16 \mathrm{fm}^{2}$ (arising entirely from Fig. 1(b)). In short, inclusion of the $\mathcal{O}\left(p^{3}\right)$ loop contribution produces a substantial cancellation with the $\mathcal{O}\left(p^{2}\right)$ term, thereby enhancing the relative sensitivity of $\left\langle r_{s}^{2}\right\rangle_{M}$ to the LEC $b_{s}^{r}$. While one generally expects a suppression of higher-order effects, the rate of convergence for the strangeness form factors is governed by $m_{K} / \Lambda_{\chi} \sim 1 / 2$ and, thus, is rather slow. Consequently, the occurance of significant cancellations between successive orders as we find here is not entirely surprising.

At this time, it is not possible to determine $\mu_{s}$ and $\left\langle r_{s}^{2}\right\rangle_{M}$ from existing measurements. As evident from Eq. (9) the strange quark vector current contains both SU(3)-octet and $\mathrm{SU}(3)$-singlet components. Contributions to to $b_{s}+0.6 b_{8}$ and $b_{s}^{r}$ from the octet component are known, while the singlet component is undetermined. In principle, one might attempt to determine $\mu_{s}$ - and, thus, $b_{s}+0.6 b_{8}$ - from the published value of $G_{M}^{(s)}\left(q^{2}=-0.1\right)$, using the lowest-order $\mathrm{HB} \chi \mathrm{PT}$ result for $\left\langle r_{s}^{2}\right\rangle_{M}$ as was done in Refs. [2, 6]. However, as the results in Eqs. (7.11) indicate, such a procedure could be misleading. Given the sensitivity of $\left\langle r_{s}^{2}\right\rangle_{M}$ to $b_{s}^{r}$, neither the magnitude nor the sign of the magnetic radius can be determined at present in a model-independent manner. Thus, the correct extrapolation of $G_{M}^{(s)}$ from $q^{2}=-0.1$ $(\mathrm{GeV} / c)^{2}$ to the photon point is simply not known at present.

To illustrate this situation, we plot in Fig. $2 G_{M}^{(s)}$ as a function of $Q^{2}=-q^{2}$, showing the published result at $Q^{2}=0.1(\mathrm{GeV} / c)^{2}$. We also show various possible extrapolations to $Q^{2}=0$. The dashed lines indicate the extrapolation for $b_{s}^{r}=-1$ and the dot-dashed lines give the extrapolation for $b_{s}^{r}=+1$. Taking these two possibilities as reasonable, though not rigorous, extremes, one would infer that the strange magnetic moment falls in 
the range $0.7 \gtrsim \mu_{s} \gtrsim-0.4$. Using only the leading order $\mathrm{HB} \chi \mathrm{PT}$ results one would infer $0.5 \gtrsim \mu_{s} \gtrsim-0.3$. We emphasize, however, that larger values for $\left|b_{s}^{r}\right|$ than used in Fig. 2 would not be unreasonable, so we would not want to use $\Delta b_{s}^{r}= \pm 1$ as an estimate of the theoretical uncertainty. For example, changing $b_{s}^{r}$ from \pm 1 to \pm 2 increases the range in $\mu_{s}$ by $\sim \pm 0.1$. We also note that, while the present uncertainty in $\mu_{s}$ is likely dominated by the experimental error, the relative importance of the $\left|b_{s}^{r}\right|$-induced uncertainty could become comparable to the experimental error with more precise data for $G_{M}^{(s)}\left(q^{2}=-0.1\right)$.

In general, LEC's such as $b_{s}$ and $b_{s}^{r}$ characterize the effects of short-distance physics arising from scales $r<1 / \Lambda_{\chi}$. Such non-perturbative effects can, in principle, be computed using lattice QCD or dispersion relations. While no definitive calculation has been performed using either approach, it is nevertheless instructive to compare the implications for the LEC's from existing analyses. In the quenched lattice calculation of Ref. [11], $G_{M}^{(s)}\left(q^{2}\right)$ was evaluated at five different kinematic points, starting at $Q^{2}=0$ and increasing by increments of roughly $\Delta Q^{2}=0.4(\mathrm{GeV} / c)^{2}$. The results at $Q^{2}=0$ give $\mu_{s}=-0.36 \pm 0.20$, corresponding to $b_{s}+0.6 b_{8} \approx-0.6 \pm 0.1$. Given both the spacing of the lattice data as well as the size of the error bars, there exists considerable lattitude for the shape of $G_{M}^{(s)}$ at low- $Q^{2}$. For example, the authors of Ref. [11] fit the lattice data to a dipole form factor, yielding a radius parameter $b_{s}^{r}(\mu=1 \mathrm{GeV})=0.23 \pm 0.13$. However, a different shape for $G_{M}^{(s)}$ at low- $Q^{2}-$ including an opposite sign slope at the origin - would not be inconsistent with the published lattice results.

In the case of dispersion relations, the strange magnetic moment and magnetic radius are related to integrals over the imaginary parts of the relevant strange form factors:

$$
\begin{aligned}
\mu_{s} & =\frac{1}{\pi} \int_{9 m_{\pi}^{2}}^{\infty} \frac{\operatorname{Im} F_{2}^{(s)}(t)}{t} d t \\
\left\langle r_{s}^{2}\right\rangle_{M} & =\frac{6}{\pi} \int_{9 m_{\pi}^{2}}^{\infty} \frac{\operatorname{Im} G_{M}^{(s)}(t)}{t^{2}} d t .
\end{aligned}
$$

Note that since the Sachs form factor $G_{M}^{(s)}\left(q^{2}\right)$ is the sum of Dirac and Pauli form factors, $F_{1}^{(s)}$ and $F_{2}^{(s)}$, respectively, and since $F_{1}^{(s)}(0)=0$, only the integral over $F_{2}^{(s)}$ contributes to $\mu_{s}$.

In each case, the integrands receive contributions from a tower of hadronic states: $3 \pi$, $5 \pi, 7 \pi, K \bar{K}, \ldots$ In previous work 12, 13, we evaluated the $K \bar{K}$ contribution using $K N$ scattering and $e^{+} e^{-} \rightarrow K \bar{K}$ data. We find that the "kaon cloud" contribution to both the strange vector form factors and EM isoscalar form factors is dominated by the $\phi(1020)$ resonance. For the purely pionic intermediate states, $e^{+} e^{-}$data suggest that only the $3 \pi$ channel gives an important contribution and that it is dominated by the $\omega$-resonance. Using the known flavor content of the $\omega$, one can follow Ref. [14 and evaluate its contribution to $G_{M}^{(s)}$.

Inclusion of higher-mass states $(e . g ., K K \pi)$ is necessary in order to obtain a physically reasonable large- $Q^{2}$ behavior of vector form factors. In the case of the isoscalar magnetic form factor, the sum of the $3 \pi$ and $K \bar{K}$ contributions - along with a single higher mass pole to approximate the effect of the remaining higher-mass states - gives an excellent description over a wide range for $Q^{2}$. Since we do not compute the higher-mass contributions 
from first principles, however, we are not confident in evaluating their contribution to $G_{M}^{(s)}$. Consequently, we quote only the "low-mass" $(3 \pi$ and $K \bar{K})$ contributions to the moments in Eqs. (12, 13): $\mu_{s}=-0.28$ and $\left\langle r_{s}^{2}\right\rangle_{M}=-0.34 \mathrm{fm}^{2}$ 13. These values correspond to $b_{s}+0.6 b_{8} \approx-0.6$ and $b_{s}^{r} \approx-1.1$, respectively. A comparison of these values with the lattice/dipole fit values is given in Table I. The corresponding low-mass dispersion relation prediction for the low- $Q^{2}$ behavior of $G_{M}^{(s)}$ is also shown in Fig. 2. A reasonable, though not rigorous, estimate for the uncertainty associated with presently unknown higher-mass contributions is also shown (shaded band)2 2 . For example, a negative slope but with smaller magnitude arises in the pole approximation analyses of form factors given in Refs. [14, 15.

We observe that the lattice and "low-mass" dispersion relation determinations of $b_{s}$ are comparable, while the dipole fit to lattice data gives a significantly different prediction for the radius parameter $b_{s}^{r}$ than obtained with the dispersion relation analysis. We speculate that this difference could be an artifact choosing a dipole form factor to parameterize the lattice evaluation of $G_{M}^{(s)}$, a result of the omission of higher-mass contributions to the dispersion integral, or both. More importantly, each of these approaches, which incorporate both chiral and nonperturbative effects, produce values for the LEC $b_{s}^{r}$ of natural size, though having opposite sign. In either case, one would conclude that non-perturbative dynamics play an important role in the physics of $G_{M}^{(s)}$. Ideally, future theoretical progress will ultimately lead to consistency between the lattice and dispersion relation treatments of these nonperturbative effects. Such progress will undoubtedly require incorporation of the correct chiral structure of $G_{M}^{(s)}$ at low- $\left|q^{2}\right|$. From the experimental side, additional data for $G_{M}^{(s)}$ at larger $\left|q^{2}\right|$ may not be sufficient to determine $\mu_{s}$ and $\left\langle r_{s}^{2}\right\rangle_{M}$, since the chiral expansion is valid for a limited $\left|q^{2}\right|$-range $\left(e . g\right.$, out to $\sim m_{K}^{2}$ ) and since new operators contribute as one moves successively further from the photon point. On the other hand, additional low$\left|q^{2}\right|$ data $\left[\right.$ e.g., $\left.\left|q^{2}\right|<0.05(\mathrm{GeV} / c)^{2}\right]$ from parity-violating electron scattering experiments could provide an unambiguous determination of the behavior of strange magnetism at small momentum-transfer.

This work was supported in part under U.S. Department of Energy contract \#DE-FGO200ER41146 and by the National Science Foundation under award PHY00-71856 and grant No. PHY-0098645. We thank B.R. Holstein, B. Kubis, R.D. McKeown, U.-G. Meißner, and T.M. Ito for useful discussions.

\footnotetext{
${ }^{2}$ Additional potential sources of uncertainty include those associated with the $\omega$-pole approximation for the $3 \pi$ contribution, the analytic continuation used to obtain the spectral functions, etc..
} 


\section{REFERENCES}

[1] See, e.g., B.W. Filippone and X. Ji, Adv. in Nucl. Phys. 26, 1 (2001) and J. Gasser, H. Leutwyler, and M.E. Sainio, Phys. Lett. B 253, 163 (1991).

[2] R. Hasty, et al., the SAMPLE Collaboration, Science 290, 2117 (2000).

[3] K. Aniol et al., the HAPPEX Collaboration, Phys. Lett. B 509, 211 (2001).

[4] Jefferson Laboratory experiment 00-006, D. Beck, spokesperson.

[5] Mainz experiment PVA4, D. von Harrach, spokesperson; F. Maas, contact person.

[6] T.R. Hemmert, U.-G. Meißner, and S. Steininger, Phys. Lett. B 437, 184 (1998); T.R. Hemmert, B. Kubis, and U.-G. Meißner, Phys. Rev. C 60, 045501 (1999).

[7] S.J. Puglia and M.J. Ramsey-Musolf, Phys. Rev. D 62, 034010 (2000).

[8] M.J. Ramsey-Musolf and H. Ito, Phys. Rev. C 55, 3066 (1997).

[9] B. Kubis and U.-G. Meißner, Nucl. Phys. A 679, 698 (2001).

[10] U.-G. Meißner and S. Steininger, Phys. Phys. B 499, 349 (1997).

[11] S.J. Dong, K.F. Liu, and A.G. Williams, Phys. Rev. D 58, 074504 (1998).

[12] M.J. Ramsey-Musolf and H.-W. Hammer, Phys. Rev. Lett. 80, 2539 (1998); H.-W. Hammer and M.J. Ramsey-Musolf, Phys. Rev. C 60, 045205 (1999).

[13] H.-W. Hammer and M.J. Ramsey-Musolf, Phys. Rev. C 60, 045204 (1999).

[14] R. L. Jaffe, Phys. Lett. B 229, 275 (1989).

[15] H.-W. Hammer, U.-G. Meißner, and D. Drechsel, Phys. Lett. B 367, 323 (1996). 
FIGURES

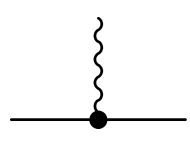

(a)

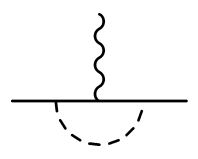

(e)

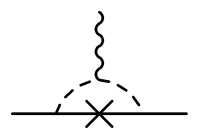

(i)

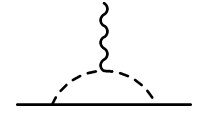

(b)

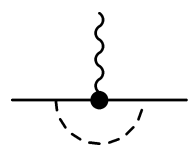

(f)

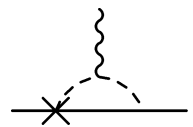

(j)

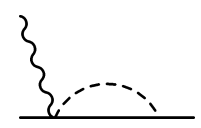

(c)

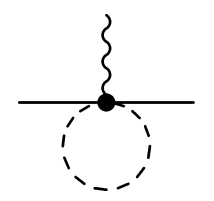

(g)

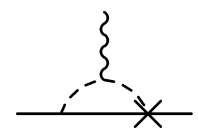

(k)

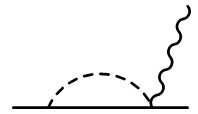

(d)

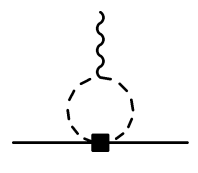

(h)

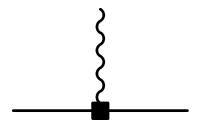

(l)

FIG. 1. Contributions to strange magnetic moment and radius. Circle denotes leading-order magnetic moment and radius operators, while square indicates operators proportional to quark mass or containing two derivatives. The $\times$ indicates a $1 / M_{N}$ insertion. Solid line and dashed lines indicate octet baryons and pseudoscalar mesons, respectively.

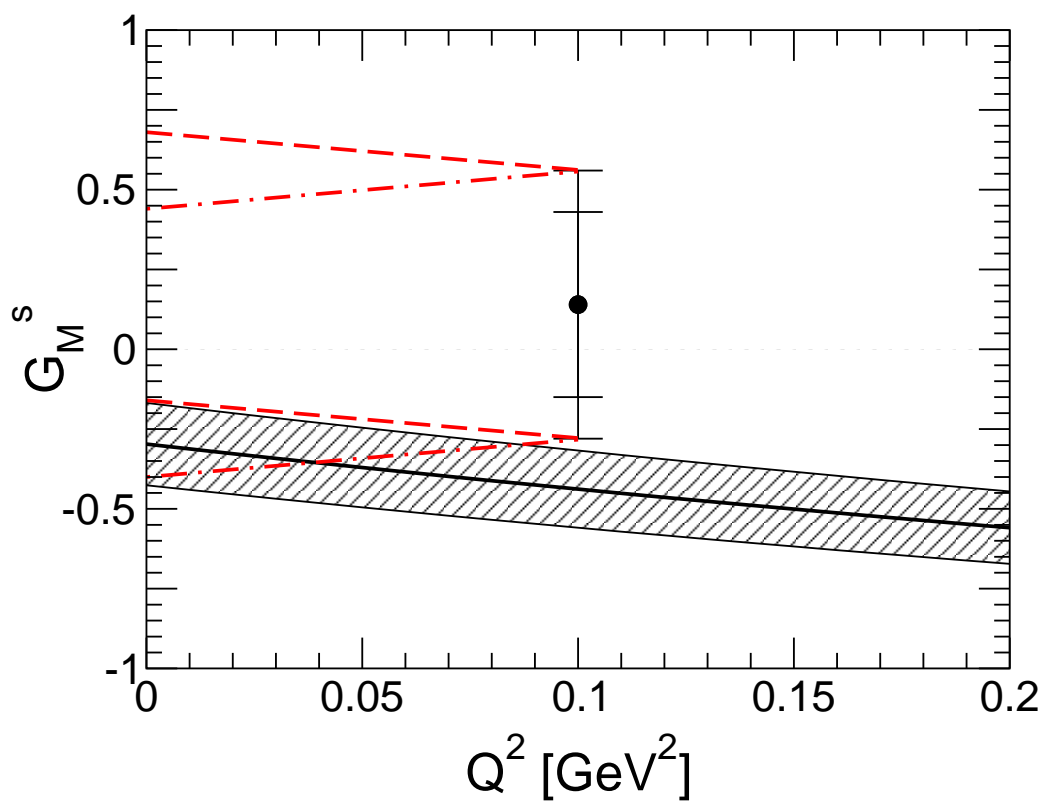

FIG. 2. The $Q^{2}$-dependence of $G_{M}^{(s)}$. Circle at $Q^{2}=0.1(\mathrm{GeV} / c)^{2}$ indicates the result from the SAMPLE measurement [2]. Inner error bar gives statistical error while outer error bar combines statistical and systematic errors in quadrature. Dashed (dot-dashed) lines indicate extrapolation to $Q^{2}=0$ using radius parameter $b_{s}^{r}=-1(+1)$. Solid line gives "low-mass" dispersion relation result [13], while shaded region indicates possible effects of higher mass contributions to the dispersion relation. 


\section{TABLES}

\begin{tabular}{|c|c|c|}
\hline \hline Method & $b_{s}+0.6 b_{8}$ & $b_{s}^{r}$ \\
\hline Lattice/Dipole Fit & $-0.6 \pm 0.1$ & $0.23 \pm 0.13$ \\
\hline Low-mass Dispersion Relation & -0.6 & -1.1 \\
\hline \hline
\end{tabular}

TABLE I. Low-energy constants for strange magnetic moment $\left(b_{s}\right)$ and strange magnetic radius $\left(b_{s}^{r}\right)$ obtained from (a) lattice QCD calculation of Ref. 11] and (b) $3 \pi$ and $K \bar{K}$ contributions using dispersion relations [13]. The the lattice value for $b_{s}^{r}$ was obtained by fitting lattice data to a dipole form for $G_{M}^{(s)}\left(q^{2}\right)$. 